\title{
Unusual shock wave in two-species driven systems with an umbilic point
}

\author{
Vladislav Popkov ${ }^{1}$ and Gunter M. Schütz ${ }^{2}$ \\ ${ }^{1}$ Max Planck Institute for Complex Systems, Nöthnitzer Straße 38, 01187 Dresden, Germany and \\ Dipartimento di Fisica e Astronomia, Università di Firenze, via G. Sansone 1, 50019 Sesto Fiorentino, Italy \\ ${ }^{2}$ Theoretical Soft Matter and Biophysics, Institute of Complex Systems II, Forschungszentrum Jülich, 52425 Jülich, Germany and \\ Interdisziplinäres Zentrum für Komplexe Systeme, Universität Bonn, Brühler Straße 7, 53119 Bonn, Germany
}

(Received 7 June 2012; revised manuscript received 10 September 2012; published 25 September 2012)

\begin{abstract}
Using dynamical Monte Carlo simulations we observe the occurrence of an unexpected shock wave in driven diffusive systems with two conserved species of particles. This $U$ shock is microscopically sharp, but does not satisfy the usual criteria for the stability of shocks. Exact analysis of the large-scale hydrodynamic equations of motion reveals the presence of an umbilical point which we show to be responsible for this phenomenon. We prove that such an umbilical point is a general feature of multispecies driven diffusive systems with reflection symmetry of the bulk dynamics. We argue that a $U$ shock will occur whenever there are strong interactions between species such that the current-density relation develops a double well and the umbilical point becomes isolated.
\end{abstract}

DOI: 10.1103/PhysRevE.86.031139

PACS number(s): 05.70.Ln, 64.60.Ht, 02.50.Ga, 47.70.-n

\section{INTRODUCTION}

Nonequilibrium lattice gas models of interacting particles with noisy dynamics [1,2] are paradigmatic models of systems far from equilibrium and find a wide range of applications in biological, social, and physical contexts [3-5]. Driving forces due to bulk fields or boundary gradients lead to steady state currents that invalidate the condition of detailed balance and give rise to remarkable features which have no equilibrium counterparts. As examples we mention boundary driven phase transitions, spontaneous symmetry breaking, and hysteresis in one spatial dimension. Particle systems with two or more conserved species exhibit particularly rich behavior [6].

The coarse grained space-time evolution of bulk-driven systems is governed by two fundamental types of excitations: shocks, which carry discontinuities, and rarefaction waves, which are continuous self-similar solutions of the hydrodynamic limit equations [7]. Various properties of the fundamental excitations such as stability, speed, and morphology are determined by a scalar or vector function which relates steady macroscopic currents to average particle densities, the so-called current-density relation. The topology of the current-density function (or surfaces, in the case of several species of particles) such as the number of extrema and saddle points determines qualitative features of the large scale dynamics and, in particular, the number and character of the different stationary phases and phase transitions that one can observe in the underlying microscopic model $[8,9]$. In this way microscopic details of local particle interactions are largely irrelevant as long as they produce a certain type of current-density relation.

In this work we identify a large-scale excitation, reminiscent of a shock wave, but which should be unstable according to usual shock stability criteria. Focusing on models with two particle species we relate its appearance to a special property of the current-density relation, the presence of an isolated umbilic point. A generic umbilic point is a point on a current-density surface where the two characteristic velocities coincide, which breaks a usually assumed strict hyperbolicity assumption [10]. For the new excitation to exist, further specifications are required: (i) these characteristic velocities must vanish at the umbilic point and (ii) this point must be isolated (see Sec. III). We shall call the new excitation an umbilic shock, or a $U$ shock. The aim of this article is to describe microscopic and macroscopic properties of the $U$ shock, and to investigate conditions for its appearance and stability. We find that such an excitation is not at all exotic and can generally be observed in bidirectional models with left-right symmetry in the hopping rates, provided that there is a sufficiently strong interaction between the particles on the adjacent lanes.

The plan of the paper is the following: In Sec. II we introduce our model and describe the $U$ shock microscopically, highlighting its difference from a usual shock. In Sec. III we discuss macroscopic current-density relations with an umbilic point which makes the existence of the $U$ shock possible. In Sec. IV we prove that bidirectional models with left-right symmetry in the hopping rates all necessarily have an umbilic point. We finish with conclusions and perspectives. The Appendix contains necessary technical details.

\section{THE MODEL AND A MICROSCOPIC $U$ SHOCK}

Our model describes particles with repulsive hard-core interactions which hop unidirectionally along two chains of $L$ sites: One chain is for right-hopping particles and another chain is for left-hopping particles. At each instant of time the system is fully described by occupation numbers $n_{k} \in\{0,1\}$ (for the right movers) and $m_{k} \in\{0,1\}$ (for the left movers). A right-moving particle at site $k$ can hop to its neighboring site $k+1$ provided it is empty, with a rate that depends on the occupancies at sites $k, k+1$ on the adjacent chain (see Fig. 1). For example, a particle hops with rate $\beta$ if the adjacent sites are both occupied, etc. For clarity of presentation and analytic simplification we shall keep only one rate $\gamma=e^{\nu}$ different from the others, setting all remaining rates to 1 ,

$$
\alpha=\beta=\varepsilon=1, \quad \gamma=e^{\nu} .
$$

Then the parameter

$$
Q=\gamma-1,
$$




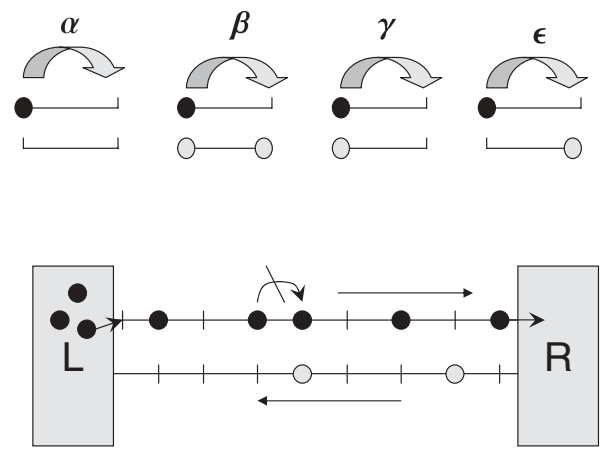

FIG. 1. Bidirectional two-chain model. For solvability, the rates must satisfy $\alpha=\beta=\varepsilon=1, \gamma=e^{\nu}$, where $v$ is the interchain interaction constant [14]. Coupling to boundary reservoirs is indicated by boxes marked $\mathrm{L}$ (the left reservoir) and $\mathrm{R}$ (the right reservoir).

which ranges from -1 to $\infty$, measures the interaction strength between the left- and right-moving species. For $Q=0$ the model reduces to two independently running totally asymmetric exclusion processes. The reason for the given choice of rates is a simplification that it offers: The currentdensity relation can be found analytically as explained in Sec. III and can therefore be analyzed in detail. For monitoring the microscopic position of shocks on the lattice we also introduce a second-class particle (SCP) [11]. Our SCP is a phantom particle, designed to track the position of a $U$ shock. Denoting the position of the SCP by $a$, the rules are

$$
\begin{array}{lll}
a \rightarrow a+1, \quad \text { if } & n_{a+1}=1, & m_{a+1}=0 . \\
a \rightarrow a-1, & \text { if } \quad n_{a-1}=0, & m_{a-1}=1 .
\end{array}
$$

This means that the SCP moves preferentially to the right (to the left) in a region with a high (low) density of right movers and low (high) density of left movers. For a $U$ shock, which connects two such regions [see Fig. 2(b)], the above dynamical rules favor positioning of the SCP at the middle of a local density gradient that corresponds to a shock on macroscopic scale. In contrast to the second-class particles in Ref. [11], our SCP does not use the sites of the chains and should rather be viewed as moving beside them, not interfering with regular particles.

The bulk dynamics for chain particles (see Fig. 1) is complemented with boundary conditions: We consider open boundaries where at the left end of the chain a right mover can enter the chain and it can leave it at the right end. Left movers are hopping to the left with the same dynamic rules. Note that in general we do not require complete left-right symmetry, so the entrance and exit rates for different species can be different. We choose a maximal feeding regime where we put a particle on the entrance site once it becomes empty and take it out from the exit site once it reaches it. The unidirectional hopping along with the open boundaries ensure that a nonzero steady state current is maintained.

Our results do not depend qualitatively on how exactly the maximal feeding regime is realized. For our dynamical Monte Carlo simulation we choose the following random sequential update procedure. For a chain of length $L$, i.e., a system of $2 L$ sites (numbered $i=1,2, \ldots, L$ for right movers and
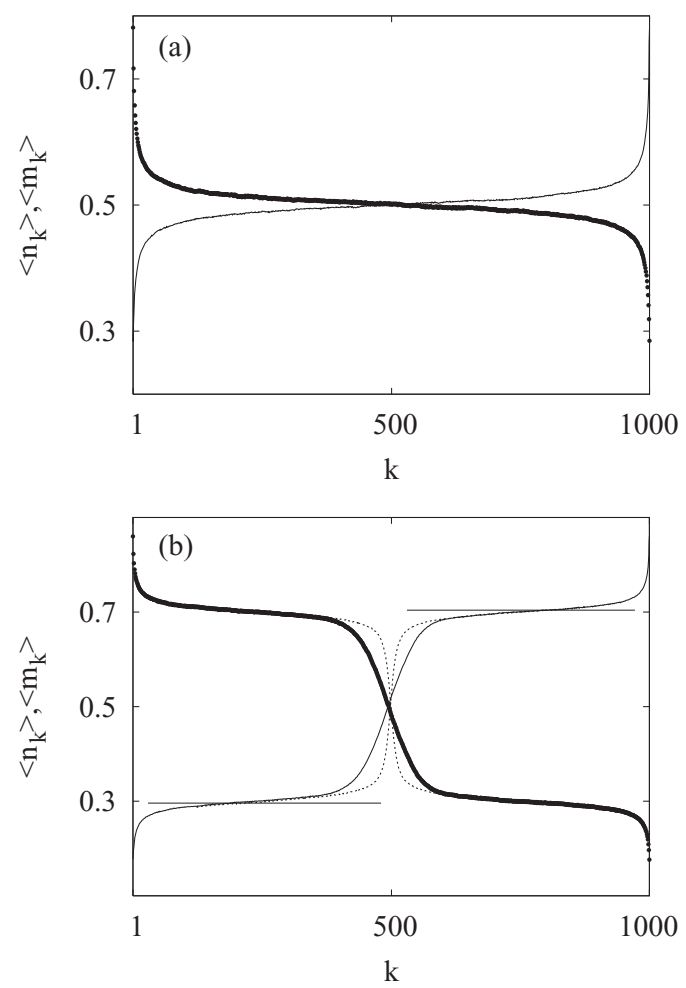

FIG. 2. Average density profiles for right movers (thick lines) and left movers (thin lines), above the phase transition $Q=-0.4$ (a) and below the phase transition $Q=-0.9$ (b). The dotted curve in (b) shows the $U$ shock viewed from a second-class particle.

$i=L+1, L+2, \ldots, 2 L$ for left movers) one Monte Carlo step consists of $2 L+2$ uniform drawings of an integer random number $s$ in the range $0 \leqslant s \leqslant 2 L+1$. If $0 \leqslant s \leqslant L$, the configuration of right movers is updated. If $s=0$, and the left boundary site $i=1$ is empty, we fill it with a particle (free entrance). If $s=L$ and the respective site contains a particle, we remove it (free exit). For intermediate $0<s<L$, if site $s$ contains a particle, a hopping is performed on the right neighboring site with given rates (1), provided it was empty. The update of the left movers is done analogously. We start from an empty lattice and after a transient time we measure site occupancies $n_{k}, m_{k}$, and take averages over many Monte Carlo steps and many histories. Typically we choose a system size up to $L=1000$ sites in each chain. The transient time for $L=1000$ is $10^{6}$ Monte Carlo steps, and averaging up to ten histories is done. We perform the measurements for different values of the interaction parameter $Q$. Note that due to the hard-core exclusion, the average densities of the right- and left-moving particles may only take values between 0 and 1 .

The maximal feeding regime usually leads to the largest particle current since we facilitate maximally the entrance and exit of particles at the boundary. In the absence of interaction $(Q=0)$ such boundary conditions lead to a state with average particle densities $1 / 2$, a state with maximal possible particle current $[12,13]$. In the presence of interaction $Q$ the stationary density profile does not undergo qualitative changes for a vast interaction range $-0.75<Q<\infty$ [see Fig. 2(a)]. However, for values of $Q<-0.75$ one observes something very unusual and different. The bulk density profile 

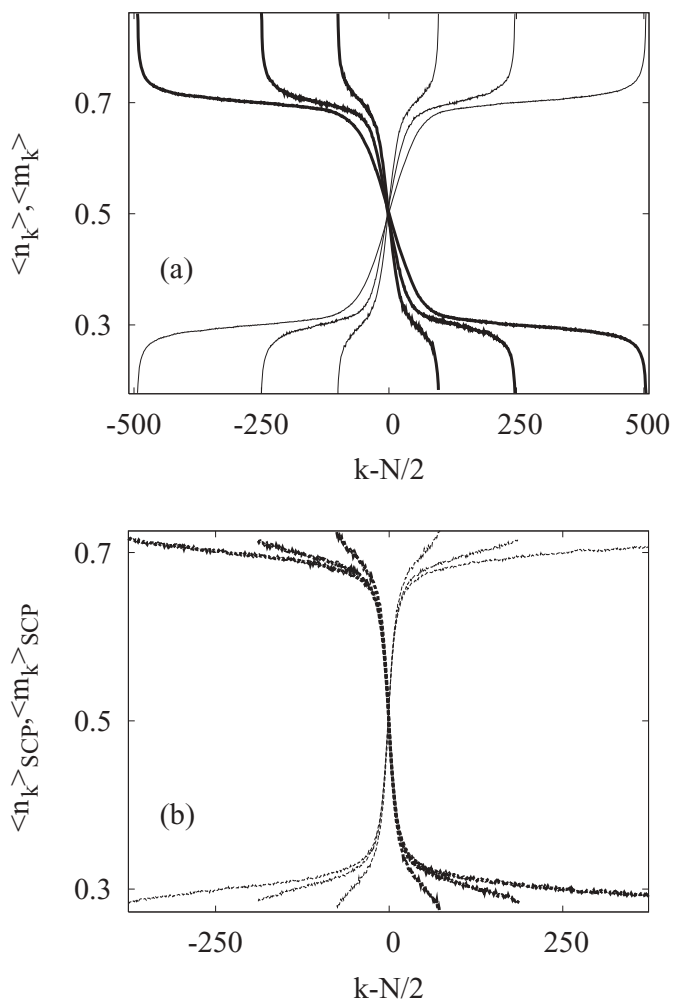

FIG. 3. (a) Average density profiles for right movers (thick lines) and left movers (thin lines), for $Q=-0.9$ and different system sizes $N=200,500,1000$. For a better view the $U$ shock is centered at the origin. (b) shows the same $U$-shock profiles, as seen from the second-class particle.

becomes inhomogeneous and consists of two plateaus with an interface in the middle. The profiles of the two species are left-right symmetric but in each plateau the densities $\rho_{1}, \rho_{2}$ of the left and right movers are different [see Fig. 2(b)]. As the interaction becomes stronger, the difference $\rho_{1}-\rho_{2}$ grows and reaches the maximum $\rho_{1}-\rho_{2}=0.5$ for the extreme case $Q=-1$. Note that the asymmetry of the profile is not a result of a spontaneous symmetry breaking since the profiles are left-right symmetric and the stationary currents of both species remain equal.

What is the nature of the observed state? The maximal feeding regime in a bulk-driven particle system usually produces steady states that are controlled by rarefaction waves which (in an infinite system) are self-similar solutions of the type $\rho(x, t)=\rho\left(\frac{x-x_{0}}{t}\right)$ (see also Appendix A). However, the interface in the middle cannot be a rarefaction wave because it does not change with time. With an increase of the system size, the interface gets wider [see Fig. 3(a)]. However, the widening is due to a fluctuation of a position of the interface, since the $U$ shock seen from a second-class particle position remains size invariant [see Fig. 3(b)]. This is a property typical of a shock.

However, the interface we observe is not a usual shock, either. In order to see this, it is instructive to look at individual particle trajectories across the interface [see Fig. 4]. Unlike the trajectories running across a shock, the particles are moving slowly (in an environment of large density) across the left side of the lattice, and then accelerate after crossing the inhomogeneity to the right-hand side of the lattice.

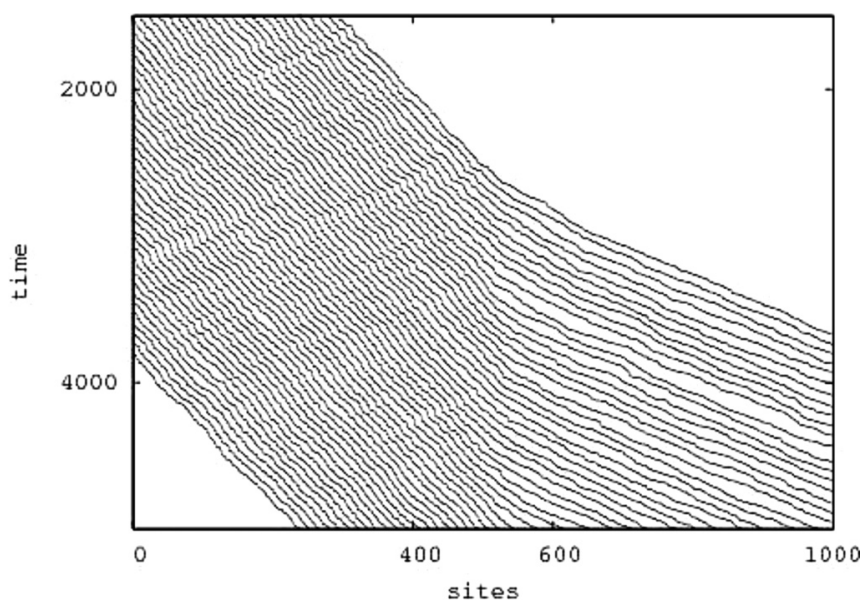

FIG. 4. Space-time trajectories of the right movers across a $U$ shock. Every tenth trajectory is shown. A system of 1000 sites was equilibrated for $2 \times 10^{6}$ Monte Carlo steps before the trajectories were recorded. The parameters are $Q=-0.9$.

Moreover, according to usual shock stability conditions, using characteristic velocities (see details in Sec. III), the interface shown in Fig. 2 should be unstable. As we shall argue below, the reason for the stability and existence of the new state is an isolated umbilic point in the current-density relation.

\section{UMBILIC POINT IN A CURRENT-DENSITY RELATION}

The model (1) that we consider has the remarkable property that the stationary distribution is a product measure [14]. The steady state probabilities of any configuration $C$ in a periodic system are given by

$$
P_{C}=Z^{-1} \prod_{k=1}^{L} e^{-v n_{k} m_{k}},
$$

where $n_{k}, m_{k}$ are particle occupation numbers on site $k$ on chains 1 and 2. With (3) the stationary currents $j_{1}$ and $j_{2}$ of the right- and left-moving species can be calculated exactly as

$$
\begin{aligned}
& j_{1}(u, v)=u(1-u)+Q \Omega_{11}(u, v) \Omega_{00}(u, v), \\
& j_{2}(u, v)=-v(1-v)-Q \Omega_{11}(u, v) \Omega_{00}(u, v),
\end{aligned}
$$

where $u$ and $v$ are the average densities of the right and left movers. The quantities $\Omega_{11}$ and $\Omega_{00}$ are stationary probabilities to have two adjacent particles and two adjacent holes, given by

$$
\Omega_{11}=\frac{(u+v-1) Q-1+\sqrt{[(u+v-1) Q-1]^{2}+4 Q u v}}{2 Q},
$$

$\Omega_{00}=1-u-v-\Omega_{11}$.

From the stationary currents we construct the flux Jacobian $(D j)$,

$$
(D j)=\left(\begin{array}{ll}
\frac{\partial j_{1}}{\partial u} & \frac{\partial j_{1}}{\partial v} \\
\frac{\partial j_{2}}{\partial u} & \frac{\partial j_{2}}{\partial v}
\end{array}\right)
$$



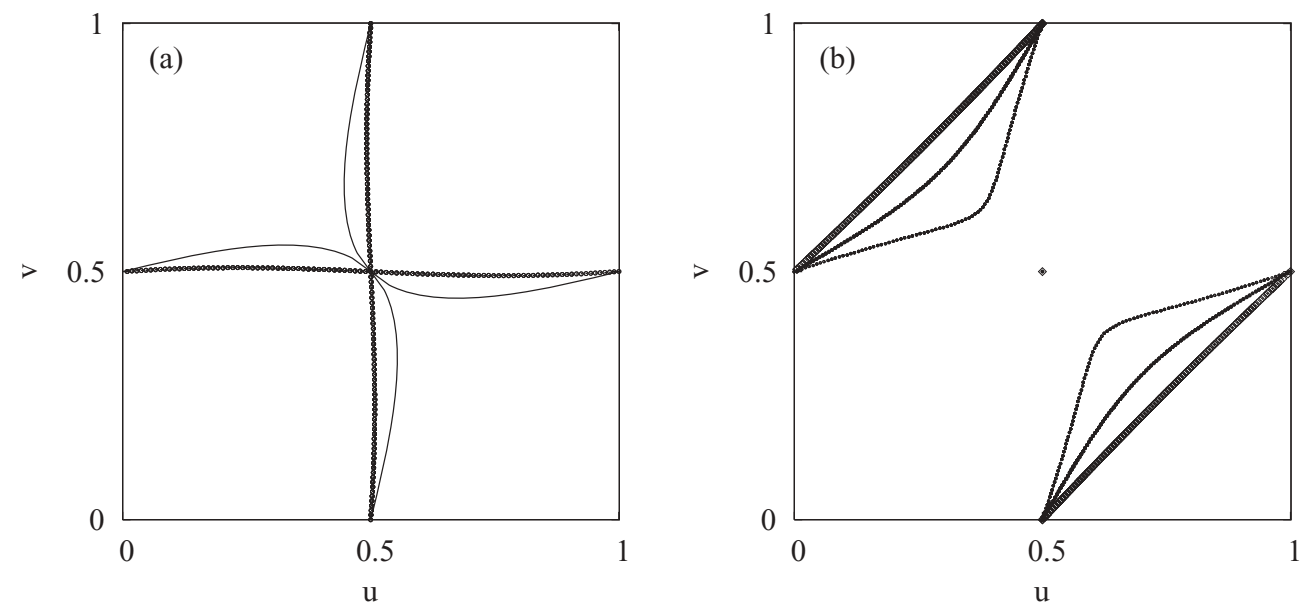

FIG. 5. Location of the curves in the $u-v$ plane where at least one characteristic velocity vanishes, $c_{i}(u, v)=0$, for small negative $Q=-0.5,-0.75[(\mathrm{a})$, thick and thin lines, respectively] and large negative $Q=-0.8,-0.9,-0.99[(\mathrm{~b})$, thin, medium, and thick lines, respectively]. The point $u=v=1 / 2$ is an umbilical point where $c_{1}=c_{2}=0$ for any value of $Q$. For $Q<-0.75$, the umbilical point becomes an isolated point.

Its two eigenvalues $c_{1,2}(u, v)$ play a fundamental role as characteristic speeds of the system of conservation laws

$$
\begin{aligned}
& \partial_{t} u+\partial_{x} j_{1}(u, v)=0, \\
& \partial_{t} v+\partial_{x} j_{2}(u, v)=0,
\end{aligned}
$$

which describes the coarse-grained dynamics on macroscopic scale. Microscopically the characteristic speeds are the velocities of the localized perturbations of a stationary homogeneous background with densities $u, v$ [14]. As such, they determine stability of shocks and rarefaction waves in the system.

A commonly made assumption about the flux functions $j_{1}, j_{2}$, called strict hyperbolicity, reads as follows: the characteristic speeds are different $c_{1}(u, v) \neq c_{2}(u, v)$ for all $u, v$. Strictly hyperbolic systems have only two types of fundamental solutions: shocks and rarefaction waves [15]. As argued in the previous section, the $U$ shock is neither a usual shock nor a rarefaction wave, so it cannot be stable in a strictly hyperbolic system.

Indeed, our system is not a strictly hyperbolic one, but it has a so-called umbilic point which is defined as a point in the $u-v$ density plane where the two characteristic velocities coincide. It is straightforwardly verified from the analytic expressions for the currents that for our system this is the case at $u^{*}=v^{*}=1 / 2$, where the two characteristic speeds $c_{1,2}^{*}$ are equal and zero for all values of $Q$.

For a full discussion of the current-density relation (4) and the associated flux Jacobian (6) we note that the points where one characteristic speed vanishes generically correspond to a family of rarefaction waves $[15,16]$ (see also the Appendix). Looking at the location of the points where at least one characteristic speed vanishes, we find two different topologies, depending on $Q$. For the interaction range $-3 / 4<Q<$ $\infty$ the umbilic point $\left(u^{*}, v^{*}\right)$ is a crossing point of the curves $c_{1}(u, v)=0$ and $c_{2}(u, v)=0$. For $-1 \leqslant Q<-3 / 4$, the umbilic point $\left(u^{*}, v^{*}\right)$ is an isolated point, and the curves $c_{k}(u, v)=0$ do not cross (see Fig. 5).

The appearance of the isolated umbilic point is a consequence of a change of topology of the current surfaces $j_{k}(u, v, Q)$ from a convex to a saddle point shape at a critical value of $Q_{\mathrm{cr}}=-3 / 4$. To understand from a microscopic perspective how this happens consider a cut of the current surface along the line $v=1-u$ for small $\gamma=Q+1 \ll 1$. At half-filling $u=v=1 / 2$ the system comes into a configuration where all adjacent sites are either both occupied or both empty and essentially gets stuck: All hoppings from this configuration are suppressed by a small hopping rate $\gamma \ll 1$. This strong interaction between the particles on the adjacent lanes explains the occurrence of a double maximum in the curve $j(u, 1-u, Q)$ for $Q<Q_{\text {cr }}$ as shown in Fig. 6, and respectively, to a saddle point on the surfaces $j_{k}(u, v, Q)$. The appearance of the saddle point then gives rise to the isolated umbilic point. The positions and amplitudes of the extrema of $g(u, Q)=j(u, 1-u, Q)$ are readily found from (4) and (5): For $Q>Q_{\text {cr }}$ the $j(u, 1-u, Q)$ curve has one maximum at $u_{0}^{*}=1 / 2$, while for $-1 \leqslant Q<Q_{\text {cr }}$ it has three extrema at positions $u_{0}^{*}=1 / 2$ and $u_{1,2}^{*}=\frac{1}{2} \pm \sqrt{3 Q^{-1}+4} / 4$.

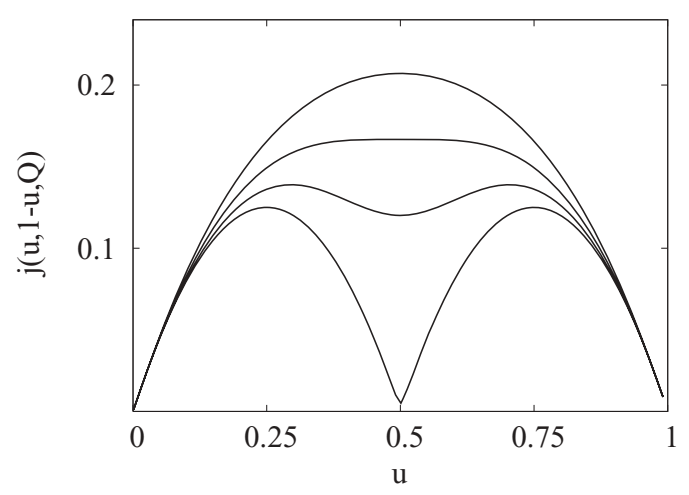

FIG. 6. Bidirectional two-chain model. Cuts of the stationary current surface along $v=1-u$ line: $j_{1}(u, 1-u, Q)$ at different $Q=-0.5,-0.75,-0.9,-0.9999$ (curves up to down). The cuts of the stationary current surface along the perpendicular direction $u=v$ remain convex for all values of $Q$ (data not shown). 
The respective currents are $j_{0}^{*}=\sqrt{Q+1} /(2 \sqrt{Q+1}+2)$ and $j_{1}^{*}=j_{2}^{*}=1 /(8|Q|)$.

Now we are in a position to analyze the $U$-shock solution. We can identify the average bulk densities of the left plateau $u_{-}, v_{-}$of the $U$ shock (see Fig. 2) with $u_{1}^{*}, u_{2}^{*}$, respectively, for the following reasons: (a) We expect the $U$-shock plateaus, piecewise, to be governed by a rarefaction wave, meaning that at least one of the characteristic speeds must vanish, $c_{i}\left(u_{-}, v_{-}\right)=0$. (b) We expect the stationary current amplitudes for right- and left-moving species to be equal, due to left-right symmetry, i.e., $j_{1}\left(u_{-}, v_{-}\right)=-j_{2}\left(u_{-}, v_{-}\right)$. We readily find, using (4) and (5), three pairs of solutions satisfying (a) and (b): (i) $\left(u_{-}, v_{-}\right)=(1 / 2,1 / 2)$, (ii) $\left(u_{-}, v_{-}\right)=\left(u_{1}^{*}, u_{2}^{*}\right)$, and (iii) $\left(u_{-}, v_{-}\right)=\left(u_{2}^{*}, u_{1}^{*}\right)$. Comparing with the $U$ shock, we find $\left(u_{-}, v_{-}\right)=\left(u_{1}^{*}, u_{2}^{*}\right)$ to be the relevant solution. Indeed solution (iii) is not compatible with our boundary conditions, while solution (i) would result in a reduction of the particle current and is dynamically unstable.

For the right plateau of the $U$ shock, we find analogously $\left(u_{+}, v_{+}\right)=\left(u_{2}^{*}, u_{1}^{*}\right)$. Apparently, the first solution (i) is unstable for $Q<Q_{\mathrm{cr}}$. So, we have for $Q<Q_{\mathrm{cr}}$,

$$
\begin{aligned}
& u_{-}(Q)=v_{+}(Q)=\frac{1}{2}+\frac{\sqrt{3 Q^{-1}+4}}{4}, \\
& v_{-}(Q)=u_{+}(Q)=\frac{1}{2}-\frac{\sqrt{3 Q^{-1}+4}}{4} .
\end{aligned}
$$

The stationary currents for the $U$ shock are then given by the $j_{k}\left(u_{1}^{*}, u_{2}^{*}, Q\right)$,

$$
j_{\text {stat }}^{U}=\frac{1}{8|Q|} \text { for } \quad Q<-\frac{3}{4} .
$$

Above the critical point, the bulk densities are $u=v=1 / 2$, and the stationary currents are given by $j_{k}(1 / 2,1 / 2, Q)$,

$$
j_{\text {stat }}=\frac{\sqrt{Q+1}}{2 \sqrt{Q+1}+2} \quad \text { for } \quad Q \geqslant-\frac{3}{4} .
$$

Our analytical predictions are well borne by the Monte Carlo simulations (see Fig. 7). Note that by establishing an inhomogeneous state (the $U$ shock) below $Q_{\text {cr }}$ the system

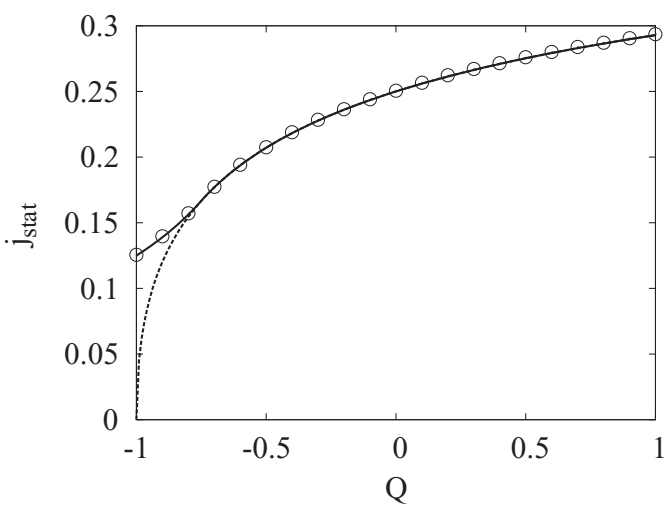

FIG. 7. Stationary currents of the bidirectional model at maximal feeding as a function of $Q$. Circles show Monte Carlo results for a system of size 600 , while the solid curve is the theoretical prediction [(10) and (11)]. Below the critical $Q$, the branch (11), indicated by the broken line, becomes unstable [(11)]. optimizes its current, which would be strongly suppressed for any symmetric bulk homogeneous state. This can be viewed as a generalization of the phenomenon of a current maximization at maximal feeding [17] to a system with two species.

We can study the phase transition in our model at $Q=Q_{\text {cr. }}$. Let us choose the difference $u_{-}(Q)-v_{-}(Q)=\Delta$ between the bulk densities of the right and left movers to be our order parameter. We have $\Delta=0$ for $Q>Q_{\text {cr }}$ and $\Delta=\frac{1}{2} \sqrt{3 Q^{-1}+4}$ for $Q<Q_{\mathrm{cr}}$. Near the transition, $\left.\Delta\right|_{Q_{\mathrm{cr}}-\delta q}=2 \sqrt{\delta q / 3}$, so we have a square root singularity, similar to that arising in the Landau theory of continuous phase transitions generated by a change of a free energy potential from a single to a double minimum topology. On the other hand, the stationary current is continuous across the transition point together with its first derivative,

$$
\left.j_{\text {stat }}\right|_{Q_{\text {cr }}-\delta q}-\left.j_{\text {stat }}\right|_{Q_{\text {cr }}}=O\left(\delta q^{2}\right), \quad \text { for } \quad \delta q \ll 1 .
$$

Finally, we comment on the robustness of the $U$ shock. The $U$ shock turns out to be very robust with respect to a choice of the boundary conditions (BC). We observe the $U$ shock for a wide choice of $\mathrm{BC}$, which, in particular, do not need to be left-right symmetric. In order to formulate the conditions for its appearance more precisely, we need to parametrize the $\mathrm{BC}$ in terms of boundary reservoirs. Such a parametrization involves further technical details [18] and is out of the scope of the present paper.

The $U$ shock is also stable with respect to a change of the model parameters, e.g., the bulk hopping parameters, as long as they remain left-right symmetric. In particular, the particle-hole symmetry of the hopping rates (1), which makes the particle current invariant with respect to $u, v \rightarrow 1-u$, $1-v$ interchange (see Fig. 6) can be relaxed without causing qualitative changes to a $U$-shock.

\section{UMBILIC POINT IN BIDIRECTIONAL MODELS}

With the system we have studied, we were lucky enough to find the stationary currents analytically and establish the existence of the umbilic point. How exotic is this point? Moreover, is it possible to predict, from the microscopic transition rates, if the system will have such a point? In this section we prove that an umbilic point with $c_{1}=c_{2}=0$ is not at all exotic and is present necessarily in bidirectional models with left-right symmetry.

Let us consider Markov processes involving two driven particle species which are biased in opposite directions, their bulk dynamics (but not necessarily their boundary dynamics) being invariant under the left-right interchange. Let us denote the average particle densities of the two species as $u, v$ and the respective currents as $j_{1}(u, v), j_{2}(u, v)$. From the left-right symmetry we have $j_{2}(u, v)=-j_{1}(v, u)$. Let us consider the line of equal densities $u=v$. Along this line the flux Jacobian (6) takes the form

$$
D j=\left(\begin{array}{lc}
a(u) & b(u) \\
-b(u) & -a(u)
\end{array}\right),
$$

with the respective eigenvalues (characteristic speeds)

$$
c_{1}(u=v), \quad c_{2}(u=v)=\mp \sqrt{a^{2}-b^{2}} .
$$


Let us assume, in addition, that we have a restriction on a number of particles which can occupy a single lattice site, i.e., that the maximally allowed density of each species is limited to the same value $\max u=\max v=\rho_{\max }$. This assumption is necessary for our subsequent analysis. In many applications such a restriction is a consequence of the hard-core exclusion interaction.

Now, let us move along the line $u=v$ from $u=0$ to the maximally allowed value $u_{\max }$ and assume without loss of generality that our process evolves on two parallel chains, $u_{\max }=v_{\max }=\rho_{\max }$. Let us call the lane with right movers lane $A$, and the lane with left movers lane $B$.

Guided by the physical meaning of the characteristic speeds as the velocities of localized perturbations of a homogeneous state [14], we deduce that a stationary state with small density of right movers on one lane and small density of left movers on another lane, attainable for $u=v \rightarrow 0$, has characteristic speeds of opposite signs in accordance with (12). In this limit the left- and right-moving species are practically uncoupled and we can attribute the positive characteristic speed to rightmoving particles $c_{A}(u=v \rightarrow 0)>0$ and the negative characteristic speed to the left-moving particles $c_{B}(u=v \rightarrow 0)<0$. At the other end of the line $u=v \rightarrow \rho_{\max }$ we have vanishing density of left-moving holes in the dense background of the right-moving particles on lane $A$, and similarly for the right-moving holes on lane $B$. Repeating the arguments for the characteristic speeds, we have $c_{A}\left(u \rightarrow u_{\max }\right)<0$ and $c_{B}\left(u \rightarrow u_{\max }\right)=-c_{A}\left(u \rightarrow u_{\max }\right)$. By continuity we deduce that there exists a point at which the characteristic speed $c_{A}$ changes sign, $c_{A}\left(u^{*}=v^{*}\right)=0$. Moreover, due to (12), the other characteristic speed at this point also vanishes, $c_{B}\left(u^{*}=v^{*}\right)=0$. The point $u^{*}=v^{*}$ where both characteristic speeds vanish is an umbilic point.

This argument establishes the existence of at least one umbilic point with $c_{1}=c_{2}=0$ for left-right symmetric bulk dynamics. Notice that the boundary conditions do not enter the argument. Hence one may impose boundary conditions that violate the left-right symmetry without destroying the umbilic point. The presence of the umbilic point is a necessary ingredient to observe a $U$ shock, which we associate with an isolated umbilic point: In order to become isolated, sufficiently strong interactions between the species leading to a saddle point topology of the current are required.

\section{CONCLUSIONS}

We have considered an open bidirectional two-component driven diffusive system with left-right symmetry for the bulk dynamics in the maximal flow regime. We havedescribed in detail a bulk inhomogeneous solution, denoted $U$ shock. This solution has many properties of a usual shock-in particular, its microscopic sharpness-but does not satisfy the usual criteria for the stability of shocks. We have computed the critical value of the interaction above which the $U$ shock exists. We have shown that the existence of the $U$ shock is due to the intrinsic presence of an isolated umbilic point with vanishing characteristic velocities. No fine tuning of the interaction strength is required above the critical value.

The $U$ shock turns out to be robust also with respect to changes in the boundary parameters, even if they violate the (necessary) left-right symmetry of the bulk dynamics. In an open system it is only necessary to maintain a stationary maximal flow regime. More generally, we proved the existence of an umbilic point with vanishing characteristic velocities in any bidirectional model with left-right symmetry of the bulk hopping rates. The presence of umbilic points crucially alters the dynamics of the system in the hydrodynamic limit (A1), giving rise to a variety of unusual solutions, called undercompressed and overcompressed shocks [10]. The $U$ shock is one such solution. The necessary condition for a $U$ shock is a sufficiently strong interchain interaction, resulting in a saddle point in the current-density surfaces.

Bidirectional models are being widely studied in the literature, in particular, in connection with the intriguing phenomenon of spontaneous symmetry breaking (SSB) [19-28]. However, we find that in most known cases the current-density relations are convex surfaces. It would be interesting to study SSB in the presence of an isolated umbilic point. Another interesting problem is to explore the full phase diagram of the open system with an umbilic point.

\section{ACKNOWLEDGMENTS}

V.P. thanks the IZKS and the University of Bonn for hospitality and acknowledges partial support by the Alexander von Humboldt Foundation and from the italian MIUR through PRIN 20083C8XFZ initiative.

\section{APPENDIX: RAREFACTION-WAVE CONTROLLED STATIONARY STATES}

The generical importance of the points where at least one of the characteristic speeds $c_{i}$ vanishes can be demonstrated by the following argument. It is well known $[15,16]$ that partial differential equations of the type

$$
\begin{aligned}
\frac{\partial \rho_{k}}{\partial t}+\frac{\partial j_{k}\left(\rho_{1}, \rho_{2}, \ldots, \rho_{K}\right)}{\partial x} & =0, \\
k & =1,2, \ldots K,
\end{aligned}
$$

where $K$ is the number of species, admit two fundamental classes of solutions: shock waves and rarefaction waves. A rarefaction wave is a self-similar solution of (A1), depending only on the ratio $\xi=\left(x-x_{0}\right) / t$, where $x_{0}$ is the position of its center, and $t>0$. We argue that in the long-time (stationary) limit $t \rightarrow \infty$ the stationary bulk density $\rho_{\text {stat }}$ generated by a rarefaction wave has zero characteristic speed $c_{p}\left(\rho_{\text {stat }}\right)=0$. Here $\rho(x, t)$ is a vector, the components of which are the density profiles $\rho_{1}(x, t), \rho_{2}(x, t), \ldots, \rho_{K}(x, t)$ of the respective species. By $\rho_{\text {stat }}$ we denote the vector with stationary bulk densities $\left\{\rho_{1}^{\text {stat }}, \rho_{2}^{\text {stat }}, \ldots, \rho_{K}^{\text {stat }}\right\}$.

We search for a solution of (A1) in the form $\rho(x, t)=h(\xi)$. Substituting in Eq. (A1), we obtain

$$
-\frac{\xi}{t} \frac{\partial h}{\partial \xi}+\frac{1}{t}(D \mathbf{j}) \frac{\partial h}{\partial \xi}=0,
$$

where the matrix $(D \mathbf{j})$ is the Jacobian of the flux $(D \mathbf{j})_{p q}=$ $\partial j_{p} / \partial \rho_{q}$. The above equation can be rewritten as

$$
(D \mathbf{j}) \frac{\partial h}{\partial \xi}=\xi \frac{\partial h}{\partial \xi} .
$$


In the limit $t \rightarrow \infty$, the scaled displacement $\xi=\left(x-x_{0}\right) /$ $t \rightarrow 0$ vanishes for any finite $x-x_{0}$, and the above equation reduces to $\left.(D j)\right|_{t \rightarrow \infty} h^{\prime}=0$. Each solution of this equation is an eigenvector of the flux Jacobian $D \mathbf{j}$ with zero eigenvalue. Consequently, the matrix $(D j)_{t \rightarrow \infty}=(D \mathbf{j})\left(\rho_{\text {stat }}\right)$ is a matrix with zero eigenvalue, i.e., at the point $\rho_{\text {stat }}$ at least one $c_{p}\left(\rho_{\text {stat }}\right)=0$. The respective rarefaction wave is called a $p$-rarefaction wave $[15,16]$. Of course, in order to guarantee the stability of the above discussed rarefaction wave with respect to local perturbations at the boundaries, the boundary conditions have to be chosen appropriately.
[1] T. M. Liggett, Stochastic Interacting Systems: Contact, Voter and Exclusion Processes (Springer, Berlin, 1999).

[2] G. M. Schütz, in Phase Transitions and Critical Phenomena, edited by C. Domb and J. Lebowitz, Vol. 19 (Academic, London, 2001)

[3] D. Mukamel, in Soft and Fragile Matter: Nonequilibrium Dynamics, Metastability and Flow, edited by M. E. Cates and M. R. Evans (Institute of Physics Publishing, Bristol, 2000).

[4] M. R. Evans and T. Hanney, J. Phys. A 38, R195 (2005).

[5] A. Schadschneider, D. Chowdhury, and K. Nishinari, Stochastic Transport in Complex Systems (Elsevier, Amsterdam, 2010).

[6] G. M. Schütz, J. Phys. A: Math. Gen. 36, R339 (2003).

[7] C. Kipnis and C. Landim, Scaling Limits of Interacting Particle Systems (Springer, Berlin, 1999).

[8] V. Popkov and G. M. Schütz, Europhys. Lett. 48, 257 (1999).

[9] V. Popkov, J. Stat. Mech. (2007) P07003; V. Popkov and M. Salerno, Phys. Rev. E 83, 011130 (2011).

[10] G.-Q. Chen and P. T. Kan, Arch. Ration. Mech. Anal. 130, 231 (1995); 160, 325 (2001).

[11] P. Ferrari, C. Kipnis, and E. Saada, Ann. Probab. 19, 226 (1991).

[12] G. Schütz and E. Domany, J. Stat. Phys. 72, 277 (1993).

[13] B. Derrida, M. R. Evans, V. Hakim, and V. Pasquier, J. Phys. A 26, 1493 (1993).

[14] V. Popkov and G. M. Schutz, J. Stat. Phys. 112, 523 (2003).
[15] P. D. Lax, Hyperbolic Partial Differential Equations, Courant Lecture Notes in Mathematics, Vol. 14 (AMS/Courant Institute of Mathematical Sciences, New York, 2000).

[16] P. D. Lax, Hyperbolic Systems of Conservation Laws and the Mathematical Theory of Shock Waves, Vol. 11 (SIAM, Philadelphia, 1973).

[17] J. Krug, Phys. Rev. Lett. 67, 1882 (1991).

[18] V. Popkov and G. M. Schütz, J. Stat. Mech. (2004) P12004.

[19] M. R. Evans, D. P. Foster, C. Godréche, and D. Mukamel, Phys. Rev. Lett. 74, 208 (1995).

[20] P. F. Arndt, T. Heinzel, and V. Rittenberg, J. Stat. Phys. 90, 783 (1998).

[21] M. Clincy, M. R. Evans, and D. Mukamel, J. Phys. A 34, 9923 (2001).

[22] S. Klumpp and R. Lipowsky, Europhys. Lett. 66, 90 (2005).

[23] D. W. Erickson, G. Pruessner, B. Schmittmann, and R. K. P. Zia, J. Phys. A 38, L659 (2005).

[24] R. D. Willmann, G. M. Schütz, and S. Großkinsky, Europhys. Lett. 71, 542 (2005); S. Großkinsky, G. M. Schütz, and R. D. Willmann, J. Stat. Phys. 128, 587 (2007).

[25] E. Pronina and A. B. Kolomeisky, J. Phys. A 40, 2275 (2008).

[26] V. Popkov, M. Evans, and D. Mukamel, J. Phys. A 41, 432002 (2008).

[27] S. Gupta, D. Mukamel, and G. M. Schütz, J. Phys. A 42, 485002 (2009).

[28] Z. D. Sun, R. Jiang, M.-B. Hu, and Q.-S. Wu, Phys. Lett. A 374, 4080 (2010). 